\title{
Decoherence dynamics of a single spin versus spin ensemble
}

\author{
V. V. Dobrovitski, ${ }^{1}$ A. E. Feiguin,,${ }^{2,3}$ D. D. Awschalom, ${ }^{4}$ and R. Hanson ${ }^{5}$ \\ ${ }^{1}$ Ames Laboratory, Iowa State University, Ames, Iowa 50011, USA \\ ${ }^{2}$ University of Maryland, College Park, Maryland 20742, USA \\ ${ }^{3}$ Microsoft Station Q, University of California, Santa Barbara, California 93106, USA \\ ${ }^{4}$ Center for Spintronics and Quantum Computation, University of California, Santa Barbara, California 93106, USA \\ ${ }^{5}$ Kavli Institute of Nanoscience Delft, Delft University of Technology, P.O. Box 5046, 2600 GA Delft, The Netherlands
}

(Received 28 March 2008; revised manuscript received 26 May 2008; published 26 June 2008)

\begin{abstract}
We study decoherence of central spins by a spin bath, focusing on the difference between measurement of a single central spin and measurement of a large number of central spins (as found in typical spin-resonance experiments). For a dilute spin bath, the single spin demonstrates Gaussian free-induction decay, in contrast to exponential decay characteristic of spin ensembles. A strong difference between a single spin and a spin ensemble also exists for the Rabi oscillation decay: for a repeated Rabi oscillation experiment, suppression of decoherence happens for a single spin while acceleration takes place for a spin ensemble. The mathematical origin of such behavior is similar to quantum Zeno/anti-Zeno effects.
\end{abstract}

DOI: 10.1103/PhysRevB.77.245212

\section{INTRODUCTION}

The ability to accurately manipulate and control single electron and/or nuclear spins holds much potential for applications in coherent spintronics, ${ }^{1}$ quantum information processing, ${ }^{2-4}$ and high-precision metrology. ${ }^{5,6}$ Also, the studies of single spins allow detailed exploration of fundamental questions related to complex quantum dynamics in spin systems. During the last few years, rapid progress in this direction leads to implementation of measurement and control of various single-spin systems, such as single electron spins localized in quantum dots, ${ }^{7-10}$ spins of impurity centers in diamond, ${ }^{11-14}$ or electron spins in $\mathrm{SiO}_{2}$ (Refs. 15 and 16).

An important aspect of the single-spin studies is the investigation of decoherence caused by interaction between the spin in question (below referred to as a central spin) and its environment. For instance, an electron spin located in a GaAs quantum dot interacts with a bath of $\mathrm{Ga}$ and As nuclear spins. Due to entanglement established between the central spin and the bath, the reduced density matrix of the central spin represents an incoherent mixture of different spin states, instead of coherent superposition. As a result, many desirable quantum properties of the central spin are lost (e.g., it becomes unsuitable for quantum computation or for use in coherent spintronic devices).

Decoherence of a central spin by a spin bath is an important process in many physically interesting systems and situations. In particular, the free-induction decay (FID) of magnetization in standard magnetic-resonance experiments (NMR or ESR) is governed by decoherence of a central spin by a bath of spins, which are coupled by dipole-dipole interactions with the central spin. In standard NMR/ESR experiments, however, the observed signal comes from an ensemble with large number of spins, which are coupled to identical or structurally similar environments. ${ }^{17-20}$ As an example, in this paper we consider a crystal containing paramagnetic impurity spins of one species, denoted below as species $S$, which are decohered by another dilute spin species $I$, with dipole-dipole coupling between $S$ and $I$ spins. One can think, for instance, of a frequently encountered situation ${ }^{17,21}$ where $S$ spins are paramagnetic centers in non-
PACS number(s): 03.65.Yz, 76.30.Mi, 03.67.Lx

magnetic crystal and $I$ spins belong to another rare species: e.g., $S$ could be the electron spin of a nitrogen-vacancy center in diamond, and $I$ spins could be a bath of ${ }^{13} \mathrm{C}$ nuclear spins, ${ }^{11,14}$ or the bath of the electron spins of nitrogen atoms (P1 centers). ${ }^{22,23}$ We assume that the $S$ spins are extremely rare (as it is the case for impurities in reasonably clean samples), so that the coupling between different $S$ spins can be neglected and every spin $S$ can be considered separately (see Fig. 1). In this case, every spin $S$ is decohered by its own bath, which consists of the $I$ spins located sufficiently close to the given $S$ spin (having noticeable dipolar coupling to the central spin), and the FID signal of $S$ spins measures the dynamics of decoherence.

In this work, we compare the decoherence dynamics as measured on a single $S$ spin with the dynamics inferred from the FID measurement of ensembles of $S$ spins. The former shows Gaussian decay (Gaussian FID line shape) while the latter demonstrates exponential decay (Lorenzian FID line shape). The difference is due to large fluctuations in the cou-
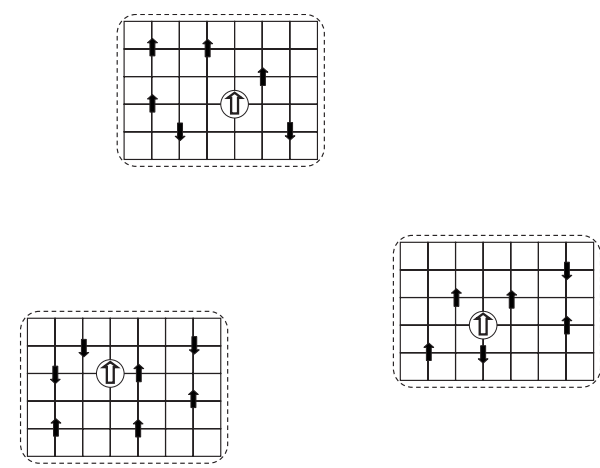

FIG. 1. Schematic representation of a crystal containing an ensemble of $S$ spins, every such spin being decohered by a bath made of dilute $I$ spins. Each window (marked by dashed line) shows a part of the crystalline sample containing an $S$ spin (white circle with white arrow inside) and a number of $I$ spins located sufficiently close to a given $S$ spin to form its own spin bath. Arrangement of $I$ spins is different for each $S$ spins, although all arrangements are structurally similar. 
pling to the environment of different $S$ spins. We show that this difference is even more pronounced in case of Rabi oscillations, when the $S$ spins are driven by strong rf field with frequency tuned in resonance with the Larmor frequency of $S$ spins. In this case, by repeating a Rabi oscillation experiment (exact protocol is described below), one can achieve suppression of decoherence for a single spin while having acceleration for spin ensemble.

We show below that the difference between the single $S$ spin and $S$-spin ensemble comes from the fluctuations in the environments made up of dilute $I$ spins due to the local arrangements (positions on the crystal lattice) of $I$ spins being different for different $S$ spins (see Fig. 1). In the mean-field terms, we can say that the dipolar fields created by the spin bath on a given $S$ spin have different distributions for each $S$ spin. It appears that these differences are very large (the second moment of the dipolar fields is diverging), despite structural similarity of environments of every $S$ spin, and are caused by strong dependence of dipole-dipole coupling on distance between two interacting spins. In contrast, such fluctuations are absent in systems with dense spin baths, e.g., in other ESR/NMR systems where the spin species $I$ are not dilute or in an ensemble of GaAs quantum dots. In these cases, local environments of every $S$ spin are exactly identical (not just similar), and for these systems, the single spin will exhibit the same decoherence pattern as an ensemble of spins.

Beside fundamental interest in comparing the decoherence dynamics of the single spin and the spin ensemble, our work may be useful for better understanding of the problems to emerge in scaling up the spintronics or quantum computation devices. Even if the device is uniform on a macroscopic scale, all elements of a large device may not be exactly the same on microscopic level, and the strength of coupling to the environment may be different for every spinbased element. Our results illustrate that, in a number of realistic situations, the fluctuations within an ensemble of similar spin-based elements may be very large, and a number of "nontypical" elements with very strong coupling to the environment may be present. While the number of such elements is small, they may qualitatively change the situation. In practice, such elements should be taken into account and controlled, and the environment of every qubit in the prospective quantum computers should be controlled at a microscopic level. Conversely, the predictions based on behavior of a large array of elements should be taken carefully: they may be too pessimistic (or too optimistic) when applied to a single "typical" element.

\section{FREE-INDUCTION DECAY}

A single $S$ spin coupled via dipolar interactions with a bath of $I$ spins is described by the Hamiltonian (everywhere below we take $\hbar=1$ );

$$
H=\omega_{0} S^{z}+\sum_{k} \omega_{k} I_{k}^{z}+\sum_{k} a_{k}\left[\mathbf{S I}_{k}-3\left(\mathbf{S n}_{k}\right)\left(\mathbf{I}_{k} \mathbf{n}_{k}\right)\right]+H_{B},
$$

where $\omega_{0}$ is the Larmor frequency of the $S$ spin, $\omega_{k}$ is the Larmor frequency of the $I_{k} \operatorname{spin}(k=1, \ldots N), \mathbf{r}_{k}$ is the vector connecting the site of $S$ spin and the site of $I_{k}$ spin, $r_{k}=\left|\mathbf{r}_{k}\right|$, $\mathbf{n}_{k}=\mathbf{r}_{k} / r_{k}$, and $a_{k}=\gamma_{S} \gamma_{I} / r_{k}^{3}$, where $\gamma_{S}$ and $\gamma_{I}$ are the gyromagnetic ratios of $S$ and $I$ spins, respectively. The term $H_{B}$ describes the dipolar interactions inside the bath. In the standard situation of large quantizing field along the $z$ axis, the Hamiltonian $H$ can be transformed to the rotating frame to give (after omitting the nonsecular terms),

$$
\tilde{H}=\sum_{k} A_{k} S^{z} I_{k}^{z}+\tilde{H}_{B},
$$

with $A_{k}=\gamma_{S} \gamma_{I}\left(1-3 n_{k}^{z}\right) /\left(2 r_{k}^{3}\right)$, where we took into account that the difference between $\omega_{0}$ and all $\omega_{k}$ is large. ${ }^{17}$

In many experimentally interesting situations, the intrabath coupling can be neglected (at least, for a certain interval of times). The spins $I_{k}$ can have $\gamma_{I} \ll \gamma_{S}$ (e.g., when $I_{k}$ are the nuclear spins and $S$ is the electron spin), in which case the intrabath dipolar coupling is small in comparison with the system-bath coupling (the latter quantified by the parameter $\left.b=(1 / 2) \sqrt{\sum_{k} A_{k}^{2}}\right)$. Also, the spread in $\omega_{k}$ can be much larger than the intrabath coupling, thus making $I_{k}^{z}$ the approximate constants of motion, ${ }^{17,18}$ in which case $\widetilde{H}_{B}$ also can be omitted. In the language of magnetic resonance, we limit ourselves to the case of $T_{2}^{*} \ll T_{2}$, where $T_{2}^{*}$ is the decay time of free-induction decay (inhomogeneous dephasing time) and $T_{2}$ is the decay time of the Hahn echo signal (homogeneous dephasing time). With the intrabath dynamics excluded, the Hamiltonian governing the FID signal has a particularly simple form;

$$
H_{0}=\sum_{k} A_{k} S^{z} I_{k}^{z}
$$

For simplicity, everywhere below we assume $S=1 / 2$ and $I_{k}$ $=1 / 2$; the calculations can be performed for any value of spins $S$ and $I$ but the answers are more cumbersome.

Initially, the $S$ spin is uncorrelated with the bath and is polarized along the $x$ axis so that its density matrix is $\rho_{S}(0)$ $=(1 / 2) \mathbf{1}_{2}+\lambda S^{x}$, where $\mathbf{1}_{2}$ is the $2 \times 2$ identity matrix and $\lambda$ is the degree of polarization (for such initial condition $\operatorname{Tr}_{S} S_{y} \rho_{S}(0)=\operatorname{Tr}_{S} S_{z} \rho_{S}(0)=0$, while $\operatorname{Tr}_{S} S_{x} \rho_{S}(0)=\lambda / 2$, where $\operatorname{Tr}_{S}$ denotes trace over the spin $S$ ). The initial state of the bath at experimentally relevant temperatures is described by the density matrix $\rho_{B}(0)=2^{-N} \mathbf{1}_{B}$, where $\mathbf{1}_{B}$ denotes the identity matrix of dimensionality $2^{N}$. The FID signal measured in magnetic resonance is proportional to the time-dependent magnetization along the $x$ axis. The latter is given by a standard quantum-mechanical average ${ }^{24}$ and has a form, ${ }^{17,18}$

$$
\begin{aligned}
M_{x}(t) & =\operatorname{Tr} S^{x} \rho(t) / \operatorname{Tr} S^{x} \rho(0) \\
& =2^{-N+1} \operatorname{Tr} S^{x} e^{-i H_{0} t} S^{x} e^{i H_{0} t} \\
& =\prod_{k} \cos \frac{A_{k} t}{2},
\end{aligned}
$$

where we normalized $M_{x}(t)$ to have $M_{x}(0)=1$. The FID line shape is given by the cosine transform of $M_{x}(t)$.

Applying the central limit theorem to this situation, ${ }^{25-28}$ one can find that the FID of a single $S$ spin is approximately Gaussian, with the width given by $b=\sqrt{\sum_{k} A_{k}^{2}}$-where the coupling parameters $A_{k}$ are determined by the positions of 
the spins $I$ on a crystal lattice with respect to the given $S$ spin. More generally, the moments of the FID line shape can be calculated directly from Eqs. (3) and (4), as demonstrated in the Appendix, giving,

$$
m_{2}=b^{2}=\sum_{k} A_{k}^{2}, \quad m_{4}=3 m_{2}^{2}-2 \sum_{k} A_{k}^{4}
$$

so that $m_{4}<3 m_{2}^{2}$ always holds; for Gaussian line shape $m_{4}$ $\approx 3 m_{2}^{2}$.

In order to calculate the FID (or FID line shape) for an ensemble of $S$ spins, one needs to average $M_{x}(t)$ over the sets of $A_{k}$, with every $S$ spin having its own set of $A_{k}$. Such a calculation is given in Ref. 18. For the systems considered here, where the $I$ spins are dilute and the FID signal decays exponentially with time, the answer is

$$
M_{x}(t)=\exp (-\Gamma t), \quad \Gamma \approx 3.8 \gamma_{I} \gamma_{S} n,
$$

where $n$ is the volume density of $I$ spins. Correspondingly, the line shape is the Lorenzian with the width $\Gamma$, and the fourth moment of the FID line $m_{4}$ is much larger than its second moment $m_{2}$ squared. ${ }^{29}$ This result remains valid (although with somewhat different numerical factors, depending on the type of underlying crystalline lattice) also for more complex situations, when the intrabath dynamics is taken into account. ${ }^{18}$

Thus, any individual $S$ spin demonstrates approximately the Gaussian FID line with $m_{4}<3 m_{2}^{2}$, while the ensemble of $S$ spins demonstrates the Lorenzian FID line with $m_{4} \gg m_{2}^{2}$. There is no contradiction here: the ensemble FID line represents an average over many central spins, each one having its own arrangement of the bath spins on a crystal lattice, and, correspondingly, its own value of $b$. The FID line shape of each single $S$ spin is Gaussian but the widths $b$ vary significantly from one central spin to another and the average over many Gaussians with different widths gives a Lorenzian line shape. Using the same approach as in Ref. 18, the distribution of the Gaussian linewidths in the ensemble of central spins can be found as

$$
P(b)=\frac{\Gamma}{b^{2}} \sqrt{\frac{2}{\pi}} \exp \left[-\Gamma^{2} /\left(2 b^{2}\right)\right],
$$

having the maximum at $b=\Gamma / \sqrt{2}$ and a very heavy powerlaw tail, ${ }^{29} 1 / b^{2}$, in the region of large $b$. This heavy tail indicates that the local distribution of $I$ spins varies very strongly from one $S$ spin to another. Such variations have been observed, e.g., in experiments on NV centers in diamond. ${ }^{13,22}$ Although the number of spins with large $b$ decreases with $b$, but "one bad apple spoils the barrel:" the effect of such rare $S$ spins with abnormally large value of $b$ (much larger than $\Gamma$ ) qualitatively changes the behavior of FID.

In practice, it means a "typical" value of $b$ for a single central spin is very weakly determined by the value of $\Gamma$. Therefore, it is impossible to reliably predict the FID time for any single central spin, and, conversely, by knowing the FID time for any single spin $S$, it is impossible to predict the
FID time for an ensemble of spins. These problems are caused by the fundamental long-range character of the dipolar interactions.

\section{RABI OSCILLATIONS}

The difference between the single $S$ spin and the ensemble of central spins becomes even more spectacular (and experimentally accessible) if we consider Rabi oscillations. The effect of the rf driving field of magnitude $H_{R}$ and frequency $\omega_{0}$ (i.e., exactly in resonance with $S$ spins) is described by adding the driving term $\gamma_{S} H_{R} S^{x} \cos \omega_{0} t$ to the Hamiltonian (1). In the rotating frame, the secular part of the Hamiltonian becomes

$$
H_{0}=h_{x} S^{x}+\sum_{k} A_{k} S^{z} I_{k}^{z}
$$

i.e., in comparison to Eq. (3), the driving term $h_{x} S^{x}$ is added, with $h_{x}=(1 / 2) \gamma_{S} H_{R}$. As above, we assume that the initial state of the bath is $\rho_{B}(0)=2^{-N} 1_{B}$, while the central spin now is assumed to be initially polarized along the $z$ axis. The Hamiltonian (8) is exactly solvable, see Refs. 25 and 26. Using the result of the Appendix, we can replace the action of the bath [in the last term of Eq. (8)] by the random static field $B$,

$$
H_{1}=h_{x} S^{x}+B S^{z},
$$

which has the Gaussian distribution of width $b$ in case of a single $S$ spin, and the Lorenzian distribution of width $\Gamma$ in case of ensemble of $S$ spins. This Hamiltonian is analytically solvable $e^{25,26}$ but the exact solution is cumbersome. In practice we are interested in the case of strong driving, when $h_{x} \gg b$ for single $S$ spin, or $h_{x} \gg \Gamma$ for an ensemble of $S$ spins. In this approximation, the evolution operator has a simple form

$$
U_{1}=\exp \left(-i S^{x} \Omega t\right), \quad \Omega=h_{x}+B^{2} /\left(2 h_{x}\right) .
$$

Then the values $M_{z}(t)$ and $M_{y}(t)$ are equal to the averages over $B$ of the expressions $\cos \Omega t$ and $-\sin \Omega t$ correspondingly.

In case of a single spin, where $B$ has a Gaussian distribution, $M_{z}(t)$ and $M_{y}(t)$ have a form of phase shifted slowly decaying oscillations (see also Refs. 25 and 26):

$$
\begin{gathered}
M_{z}(t)=M_{G}^{\mathrm{env}}(t) \cos \left(h_{x} t+\phi\right), \\
M_{y}(t)=-M_{G}^{\mathrm{env}}(t) \sin \left(h_{x} t+\phi\right), \\
M_{G}^{\mathrm{env}}(t)=\frac{1}{2}\left[1+\frac{b^{4} t^{2}}{h_{x}^{2}}\right]^{-1 / 4}
\end{gathered}
$$

where the phase shift is $\phi=(1 / 2) \arctan \left(b^{2} t / h_{x}\right)$, and $M_{G}^{\text {env }}(t)$ is the oscillations envelope (subscript " $G$ " denotes Gaussian distribution of $B$ ). It is important to note that the initial decay of the oscillations envelope, at times $2 \pi / h_{x} \ll t \ll h_{x} / b^{2}$, is quadratic,

$$
M_{G}^{\mathrm{env}}(t)=1-A_{1} t^{2}+O\left(t^{3}\right),
$$

where $A_{1} \propto b^{4}$. 
In case of an ensemble of $S$ spins, we should start again from Eq. (10) and calculate $M_{z}(t)$ and $M_{y}(t)$ for the Lorenzian distribution of $B$. The averaging over $B$ can be performed using the Fourier transform of the Lorenzian to give $M_{z}(t)=\operatorname{Re} F(t)$ and $M_{y}(t)=-\operatorname{Im} F(t)$, where

$$
F(t)=\exp \left[i h_{x} t-i \frac{\Gamma^{2} t}{2 h_{x}}\right] \operatorname{erfc}\left(\Gamma \sqrt{\frac{t}{2 h_{x}}} \frac{1-i}{\sqrt{2}}\right),
$$

and $\operatorname{erfc}(z)=1-(2 / \sqrt{\pi}) \int_{0}^{z} \exp \left(-t^{2}\right) d t$. In contrast to the single-spin case, the initial decay of the oscillations envelope, at times $2 \pi / h_{x} \ll t \ll h_{x} / \Gamma^{2}$, has a square-root form, i.e.,

$$
M_{L}^{\text {env }}(t)=1-A_{2} \sqrt{t}+O(t) .
$$

Note that this result cannot be obtained by averaging Eq. (12) over the distribution Eq. (7), since $A_{1} \propto b^{4}$ diverges in this limit. This divergence demonstrates that the short-time asymptotic expansion (12) is not valid for an ensemble of $S$ spins.

Quadratic decay has an interesting implication in quantum mechanics; it leads to the quantum Zeno effect ${ }^{30}$ (for recent developments see, e.g., Refs. 31 and 32): when a quantum system, evolving under some unitary dynamics, is subjected to quickly repeated projective measurements, the system's evolution becomes frozen. Since the decay of Rabi oscillations in our case is also quadratic in time, a similar suppression of decoherence dynamics can be observed in Rabi oscillations of a single central spin. Note, however, that this similarity should be taken carefully: the suppression of the Rabi oscillations decay in our case is not associated with any projective quantum measurement, and takes place in an open system subjected to decoherence.

The specific protocol for the repeated Rabi experiment is as follows: Let us assume that the Rabi driving field is applied for some short interval of time $2 \pi / h_{x} \ll \tau \ll h_{x} / b^{2}$, which is adjusted to be proportional to the oscillations period, so that by the time $\tau$ the central spin performed an integer number of revolutions around the $x$ axis. Then, we let the system and the bath equilibrate by waiting for the time of order of $T_{2}$ : during this time, the value of $M_{z}$ is conserved (we assume that the energy relaxation time $T_{1}$, in NMR literature often called "longitudinal relaxation time," is very long and can be taken as infinite). Then, we apply the driving field again for the interval $\tau$, wait, etc., until a large number $K$ of the Rabi field pulses is applied. At the end of the experiment, we measure $M_{z}$ (if projective measurement is used, then the whole experiment with $K$ Rabi pulses should be repeated to build up statistics). After a large number $K$ of Rabi pulses, the value of $M_{z}$ can be found by iterating formula (12);

$$
M_{z}(t)=\left(1-A_{1} \tau^{2}\right)^{K} \approx \exp \left(-A_{1} K \tau^{2}\right),
$$

where $t=K\left(\tau+T_{2}\right)$. In the limit of $K \tau=T=$ const and $K \rightarrow \infty$, the central spin demonstrates the behavior similar to Zeno effect: it becomes frozen in the state "up."

For an ensemble of central spins, for the same experiment, the situation is opposite. By iterating Eq. (14) we get,

$$
M_{z}(t)=\left(1-A_{2} \sqrt{\tau}\right)^{K} \approx \exp \left(-A_{2} K \sqrt{\tau}\right) .
$$

In the same limit of $K \tau=T=$ const and $K \rightarrow \infty$, the ensemble of central spins demonstrates an acceleration of decoherence similar to anti-Zeno behavior: the smaller $\tau$ is, the faster $M_{z}(t)$ decays.

Obviously, our conclusion is not limited to the case of $S$ $=1 / 2$ and $I=1 / 2$. Considering the derivation above, one can see that our results hold for larger spins and for more complex situations. The only essential limitations are the following: First, the whole time of the experiment should be much smaller than $T_{1}$. This implies that $T_{2} \ll T_{1}$. This is the case for a wide variety of experimental systems. Second, the time $\tau$ should be much shorter than $T_{2}$; since the only limitation on this quantity is that $\tau \gg 1 / h_{x}$, this condition can also be satisfied in many experimental systems using sufficiently strong driving fields. Third, the FID time $T_{2}^{*}$ should be much smaller than $T_{2}$; again this is the case for many experimental systems.

The study above provides a good demonstration of one of possible problems encountered in scaling up quantum systems: the fact that the bath spins are distributed uniformly on macroscopic scale does not guarantee their uniform distribution on microscopic scale. As Eq. (7) shows, in an ensemble of spins, even with structurally similar environments, there will be a sufficient number of $S$ spins with very large $b$, i.e., there will be qubits with extremely strong coupling to environment, orders of magnitude stronger than the coupling of a "typical qubit." To avoid such fluctuations, the environment of every qubit should be controlled at the microscopic level, or qubits with extreme coupling to the environment should be identified and not used for information processing.

The results above may also be of interest for better understanding of the problems emerging in controlling one central spin in comparison to the ensemble of central spins. While Rabi oscillations do not constitute a full-fledged quantum control, an interesting question arises, whether similar differences are present in the situation when the trains of pulses are applied to the single central spin and to the ensemble of spins. To study this case in realistic settings, dynamics of the bath (i.e., coupling between the bath spins) should be explicitly taken into account; this question may be answered in the future studies.

\section{ACKNOWLEDGMENTS}

We would like to thank G. Ortiz for the useful suggestions on the manuscript. Work at Ames Laboratory was supported by the Department of Energy-Basic Sciences under Contract No. DE-AC02-07CH11358. We acknowledge support from AFOSR (D.D.A.), FOM, and NWO (R.H.). A.E.F. acknowledges support from the Microsoft Corporation.

\section{APPENDIX}

To calculate $M_{x}(t)$ directly from Eqs. (3) and (4), the trace over all the states of the system in Eq. (4) is separated into the trace over the states of the $S$ spin $\operatorname{Tr}_{S}$ and the trace over the states of the bath $\operatorname{Tr}_{B}$. To calculate the latter, we note that 
all $I_{k}^{z}$ operators commute with the Hamiltonian $H_{0}$, and evaluation of $\operatorname{Tr}_{B}$ can be conveniently performed in the basis of eigenstates of the operators $I_{k}^{z}$. Thus, we take the basis wave functions of the bath as product states of individual bath spins, $|p\rangle=\left|p_{1}, p_{2}, \ldots, p_{N}\right\rangle$, where $\left|p_{k}\right\rangle$ is the eigenstate of $I_{k}^{z}$ with the eigenvalue $p_{k}$ (i.e., $\left|p_{k}\right\rangle$ is $|\uparrow\rangle_{k}$ for $p_{k}=+1 / 2$, and $\left|p_{k}\right\rangle$ is $|\downarrow\rangle_{k}$ for $\left.p_{k}=-1 / 2\right)$. Introducing the operator $\hat{B}$ $=\sum_{k} A_{k} I_{k}^{z}$ and noticing that every $|p\rangle$ is an eigenstate of $\hat{B}$, we obtain:

$$
\begin{aligned}
\operatorname{Tr}_{B} S^{x} e^{-i H_{0} t} S^{x} e^{i H_{0} t} & =\sum_{p}\left\langle p\left|S^{x} \exp \left(-i S^{z} \hat{B} t\right) S^{x} \exp \left(i S^{z} \hat{B} t\right)\right| p\right\rangle \\
& =\sum_{p} S^{x} \exp \left(-i S^{z} B_{p} t\right) S^{x} \exp \left(i S^{z} B_{p} t\right),
\end{aligned}
$$

where $\Sigma_{p}$ is the sum over all $2^{N}$ bath states $|p\rangle$, and the quantity $B_{p}=\Sigma_{k} A_{k} p_{k}$ is the eigenvalue of $\hat{B}$ corresponding to the eigenstate $|p\rangle$.

The last equation can be reinterpreted as follows: we can imagine that the spin $S$ is subjected to a random static classical field $B=\sum_{k} A_{k} p_{k}$, where $p_{k}$ are the random numbers taking the values $\pm 1 / 2$ with equal probability. Then, the trace over the bath states is equivalent to averaging over the random field $B$. Note that this is not an approximation, but a rigorous mathematical result. However, it should not be taken literally: the field $B$ is a fictitious mathematical construct, which is convenient and which considerably simplifies the calculation in more complex cases (e.g., when driving Rabi field is applied to the spin $S$ ). Decoherence is purely quantum phenomenon: it takes place due to quantum en- tanglement of the system and the bath. ${ }^{33}$ Mapping of the original quantum spin bath, with dynamically evolving system-bath correlations, onto a classical random field $B$ is possible only for a limited class of problems (with so-called "nonbranching" evolution). In general, the initial state of the bath described by the density matrix $\rho_{B}(0)=2^{-N} 1_{B}$ does not necessarily represent an equiprobable statistical mixture of all basis states $|p\rangle$ : for instance, such a matrix can correspond to the situation when the bath spins are entangled with some other environment (e.g., phonons). Interpretation of the bath density matrix is not a subject of this work, and many questions in this area are still unsolved. To avoid discussions, in this paper we consider the field $B$ as a purely mathematical construct, without ascribing any physical meaning to it.

The statistical properties of the fictitious field $B$ determine the FID line shape, e.g., it is easy to see that the moments of the FID line shape are given by the moments of the distribution of $B$. In particular (see the main text), if we consider a single $S$ spin, the distribution function of the field $B$ in the limit of large number of bath spins is determined by the central limit theorem to be Gaussian;

$$
P(B)=\frac{1}{\sqrt{2 \pi b^{2}}} \exp \left[-B^{2} /\left(2 b^{2}\right)\right],
$$

with $b=(1 / 2) \sqrt{\sum_{k} A_{k}^{2}}$. If we consider an ensemble of $S$ spins, the field $B$ should be averaged over all possible values of $b$ corresponding to all possible values of $S$ spins, see Eq. (7), which gives the Lorenzian distribution,

$$
P(B)=\frac{\Gamma}{\pi} \frac{1}{B^{2}+\Gamma^{2}} .
$$

${ }^{1}$ Semiconductor Spintronics and Quantum Computations, edited by D. Awschalom, D. Loss, and N. Samarth (Springer-Verlag, Berlin, 2002).

${ }^{2}$ D. Loss and D. P. DiVincenzo, Phys. Rev. A 57, 120 (1998).

${ }^{3}$ B. E. Kane, Nature (London) 393, 133 (1998).

${ }^{4}$ A. Imamoglu, D. D. Awschalom, G. Burkard, D. P. DiVincenzo, D. Loss, M. Sherwin, and A. Small, Phys. Rev. Lett. 83, 4204 (1999).

${ }^{5}$ L. K. Thomsen, S. Mancini, and H. M. Wiseman, Phys. Rev. A 65, 061801(R) (2002).

${ }^{6}$ J. M. Geremia, J. K. Stockton, and H. Mabuchi, Phys. Rev. Lett. 94, 203002 (2005).

${ }^{7}$ E. A. Laird, C. Barthel, E. I. Rashba, C. M. Marcus, M. P. Hanson, and A. C. Gossard, Phys. Rev. Lett. 99, 246601 (2007).

${ }^{8}$ K. C. Nowack, F. H. L. Koppens, Yu. V. Nazarov, and L. M. K. Vandersypen, Science 318, 1430 (2007).

${ }^{9}$ R. Hanson, L. P. Kowenhoven, J. R. Petta, S. Tarucha, and K. L. M. Vandersypen, Rev. Mod. Phys. 79, 1217 (2007).

${ }^{10}$ M. V. Gurudev Dutt, J. Cheng, B. Li, X. Xu, X. Li, P. R. Berman, D. G. Steel, A. S. Bracker, D. Gammon, S. E. Economou, R. B. Liu, and L. J. Sham, Phys. Rev. Lett. 94, 227403 (2005).

${ }^{11}$ J. Wrachtrup and F. Jelezko, J. Phys.: Condens. Matter 18, S807 (2006).
${ }^{12}$ T. Gaebel, M. Domhan, I. Popa, C. Wittmann, P. Neumann, F. Jelezko, J. R. Rabeau, N. Stavrias, A. D. Greentree, S. Prawer, J. Meijer, J. Twamley, P. R. Hemmer, and J. Wrachtrup, Nat. Phys. 2, 408 (2006).

${ }^{13}$ R. Hanson, F. M. Mendoza, R. J. Epstein, and D. D. Awschalom, Phys. Rev. Lett. 97, 087601 (2006).

${ }^{14}$ L. Childress, M. V. Gurudev Dutt, J. M. Taylor, A. S. Zibrov, F. Jelezko, J. Wrachtrup, P. R. Hemmer, and M. D. Lukin, Science 314, 281 (2006).

${ }^{15}$ D. Rugar, R. Budakian, H. J. Mamin, and B. W. Chui, Nature (London) 430, 329 (2004).

${ }^{16}$ R. Budakian, H. J. Mamin, and D. Rugar, Phys. Rev. Lett. 92, 037205 (2004).

${ }^{17}$ C. P. Slichter, Principles of Magnetic Resonance (SpringerVerlag, Berlin, 1990).

${ }^{18}$ A. Abragam, Principles of Nuclear Magnetism (Oxford University Press, New York, 1961). For calculation of the line shape in presence of dilute spins, see Secs. IV.IV.A and IV.IV.B.

${ }^{19}$ A. M. Tyryshkin, S. A. Lyon, A. V. Astashkin, and A. M. Raitsimring, Phys. Rev. B 68, 193207 (2003).

${ }^{20}$ W. M. Witzel, R. de Sousa, and S. Das Sarma, Phys. Rev. B 72, 161306(R) (2005).

${ }^{21}$ A. Schweiger and G. Jeschke, Principles of Pulse Electron Para- 
magnetic Resonance (Oxford University Press, New York, 2001).

${ }^{22}$ R. Hanson, V. V. Dobrovitski, A. E. Feiguin, O. Gywat, and D. D. Awschalom, Science 320, 352 (2008).

${ }^{23}$ T. A. Kennedy, J. S. Colton, J. E. Butler, R. C. Linares, and P. J. Doering, Appl. Phys. Lett. 83, 4190 (2003).

${ }^{24}$ A. Messiah, Quantum Mechanics (Interscience, New York, 1961).

${ }^{25}$ V. V. Dobrovitski, H. A. De Raedt, M. I. Katsnelson, and B. N. Harmon, arXiv:quant-ph/0112053 (unpublished); J. Sci. Eng. 1, 586 (2005).

${ }^{26}$ F. M. Cucchietti, J. P. Paz, and W. H. Zurek, Phys. Rev. A 72, 052113 (2005).

${ }^{27}$ A. V. Khaetskii, D. Loss, and L. Glazman, Phys. Rev. Lett. 88, 186802 (2002).

${ }^{28}$ I. A. Merkulov, Al. L. Efros, and M. Rosen, Phys. Rev. B 65, 205309 (2002).
${ }^{29}$ This Lorenzian is cut off at large frequencies, of orders of $\gamma_{S} \gamma_{I} / a^{3}$ (where $a$ is the lattice constant or, more generally, the minimum possible distance between the $I$ and $S$ spins). However, since the $I$ spins are diluted, $n \ll 1 / a^{3}$, this cutoff is far beyond the range of frequencies considered here and can be taken as infinity. Correspondingly, the exponential decay [Eq. (6)] of $M_{x}(t)$ sets in after some short transition time (of order of $\left.a^{3} / \gamma_{S} \gamma_{I}\right)$, but this short time scale is negligible for our purposes. In the same way, the distribution (7) has a cutoff at very large $b$, of orders of $\gamma_{S} \gamma_{I} / a^{3}$, which can be neglected at the energy/time scales considered in our work.

${ }^{30}$ J. von Neumann, Mathematical Foundations of Quantum Mechanics (Princeton University Press, Princeton, 1955).

${ }^{31}$ A. G. Kofman and G. Kurizki, Nature (London) 405, 546 (2000).

${ }^{32}$ D. Klauser, W. A. Coish, and D. Loss, arXiv:0802.2463 (unpublished).

${ }^{33}$ W. H. Zurek, Rev. Mod. Phys. 75, 715 (2003). 